\title{
Efecto terapéutico del extracto etanólico de Erythroxylum coca spp. en anemia ferropénica inducida en ratas Holtzman macho
}

\author{
Therapeutic effect of Erythroxylum coca spp. ethanol extract in \\ experimental iron-deficiency anemia in male Holtzman rats
}

\author{
Evelyn F. Gonzales-Carazas ${ }^{1,3}$, Giannina C. Melgarejo-García ${ }^{1,3}$, \\ Lizeth K. Chávez-Conde ${ }^{1,3}$, Luis J. Arellán-Bravo1, Elena Carbajal-Lázaro ${ }^{1,2}$, \\ Yasmine A. Cabrera-Gómez¹, Gilmer M. Quiróz-Sáenz¹, Isis N. García-Antúnez', \\ Miguel Llanto-Aguirre', Franchesca G. Choque-Nolasco ${ }^{1}$ \\ Estudiante de Medicina, Facultad de Medicina San Fernando, Universidad Nacional Mayor de San Marcos, Lima, Perú. \\ ${ }^{2}$ Miembro, Sociedad Científica San Fernando (SCSF), Lima, Perú. \\ ${ }^{3}$ Miembro, Asociación para el Desarrollo de la Investigación Estudiantil en Ciencias de la Salud (ADIECS-UNMSM), Lima, Perú.
}

\begin{abstract}
Resumen
Introducción: La hoja de coca ha sido usada tradicionalmente con fines medicinales y contiene altos niveles de hierro. Objetivos: Determinar el efecto del extracto etanólico de Erythroxylum coca spp. frente a anemia ferropénica inducida por dieta deficiente en hierro, en ratas Holtzman macho. Diseño: Experimental. Lugar: Laboratorio del Instituto de Patología, Facultad de Medicina, Universidad Nacional Mayor de San Marcos. Material biológico: Dieciocho ratas Holtzman macho de 16 días de edad recién destetadas. Intervenciones: Se formó tres grupos de seis ratas cada uno: a) grupo hierro suficiente (HS), recibió $25 \mathrm{~g} / \mathrm{d}$ de alimento balanceado durante 7 semanas; b) grupo hierro deficiente (HD), recibió $25 \mathrm{~g} / \mathrm{d}$ de dieta ferropénica durante 7 semanas; $y, \mathrm{c}$ ) el grupo hierro deficiente - extracto E. coca (HD-EC), recibió $25 \mathrm{~g} / \mathrm{d}$ de dieta ferropénica durante 7 semanas y a partir de la semana 5 se agregó 18 g/d de extracto de E. coca. Principales medidas de resultados: Nivel sérico de hemoglobina, peso y talla. Resultados: Al finalizar el tratamiento, se observó aumento significativo de la hemoglobina en el grupo HD-EC $(p=0,04)$. Se encontró diferencia significativa en los niveles séricos de hemoglobina entre los grupos HD-EC y HD ( $p=0,0062)$. No se encontró diferencia significativa en los valores de hemoglobina entre los grupos HD-EC y HS $(p=0,06)$. No se evidenció diferencia en el peso y la talla entre los grupos HD y HD-EC $(p=0,20$ y $p=0,23$, respectivamente). Conclusiones: $E$. coca presenta efecto antianémico experimental, sustentado en los resultados de los niveles de hemoglobina.
\end{abstract}

Palabras clave: Coca, anemia ferropénica, hemoglobina, ratas Holtzman.

\begin{abstract}
Introduction: Coca leaf has been traditionally used for medical purposes and contains high iron levels. Objective: To determine the effect of Erythroxylum coca spp. ethanol extract in iron-deficiency anemia induced by iron-deficient diet in male Holtzman rats. Design: Experimental. Setting: Institute of Pathology Laboratory, Universidad Nacional Mayor de San Marcos. Biologic material: Eighteen weaned male Holtzman rats 16 days old. Interventions: Rats were allocated to three groups of six rats each: a) iron-sufficient group (IS) received $25 \mathrm{~g} / \mathrm{d}$ of balanced diet for 7 weeks; b) iron-deficient group (ID) received $25 \mathrm{~g} / \mathrm{d}$ of iron-deficient diet for 7 weeks; and, c) irondeficient/E. coca extract group (ID-CE) received $25 \mathrm{~g} / \mathrm{d}$ of iron-deficient diet for 7 weeks, and in the fifth week $18 \mathrm{~g} / \mathrm{d}$ of $E$. coca extract were added. Main outcome measures: Serum hemoglobin level, weight and height. Results: At the end of the treatment, there was significant difference of hemoglobin in the ID-CE group $(p=0.04)$. There was significant difference in serum hemoglobin levels between ID-CE and ID groups $(p=0.0062$ ). No significant difference was found in serum hemoglobin levels between ID-CE and IS groups $(p=0.06)$. No significant difference was found in weight and height between ID and ID-CE groups ( $p=0.20$ and $p=0.23$, respectively). Conclusions: E. coca had experimental antianemic effect supported by findings in the serum hemoglobin levels.
\end{abstract}

Key words: Coca; anemia, iron-deficiency; hemoglobin; rats, Holtzman.

An Fac med. 2013;74(1):7-10 


\section{INTRODUCCIÓN}

La anemia es la concentración de hemoglobina $(\mathrm{Hb})$ por debajo del umbral establecido según edad y género ${ }^{(1,2)}$. Constituye uno de los problemas de salud con gran impacto a nivel mundial (3). La población más vulnerable es la de niños preescolares y las madres gestantes de países en vías de desarro1lo ${ }^{(4-6)}$. La prevalencia de anemia en el Perú en niños y niñas menores de cinco años es 30,7\% (ENDES 2011) (7). Es una enfermedad de etiología multifactorial, la ingesta insuficiente de hierro es la causa principal en el Perú (MINSA, Perú) ${ }^{(8)}$.

Reviste importancia las consecuencias deletéreas en el ser humano; principalmente en la población infantil. Ocasiona lesiones irreversibles a nivel neurológico, conductual, inmunológico, endocrino y somático, provocando menor crecimiento y puede predisponer a enfermedades infecciosas ${ }^{(9)}$.

Diversas medidas de prevención y tratamiento han sido planteadas en base al conocimiento de la fisiopatología de la anemia ferropénica ${ }^{(10,11)}$. No obstante, algunos tratamientos convencionales pueden producir efectos colaterales, lo cual disminuye la adherencia al tratamiento y el fracaso terapéutico ${ }^{(12)}$. Por ello, la búsqueda de alternativas terapéuticas de origen natural ${ }^{(13)}$.

La hoja de coca (Erythroxylum coca spp.) es una planta nativa de América del Sur, considerada sagrada en las ceremonias incas. Es utilizada tradicionalmente desde la época precolombina por su significado religioso y medicinal ${ }^{(14)}$. Estudios realizados por Duke y Plowman revelan que la hoja de coca contiene nutrientes, como proteínas, carbohidratos, fibra, calcio, hierro, fósforo, vitamina A y riboflavina, que satisfarían los requerimientos dietéticos del ser humano ${ }^{(15,16)}$. Además, Collazos y col. y Duke J y col. señalan concentraciones de aproximadamente $7 \mathrm{mg}$ de hierro y $6,47 \mathrm{mg}$ de vitamina $\mathrm{C}$ por cada $100 \mathrm{~g}$ de hoja de coca ${ }^{(16,17)}$.
Sobre la base de sus altas concentraciones de hierro y vitamina $\mathrm{C}$, el objetivo del presente trabajo fue evaluar el efecto del extracto etanólico de Erythroxylum coca spp. frente a anemia ferropénica inducida por dieta deficiente en hierro en ratas Holtzman macho.

\section{MÉTODOS}

Se realizó un estudio experimental con una muestra de 18 ratas Holtzman macho ${ }^{(18)}$ de 16 días de edad, recién destetadas ${ }^{(19)}$, con un peso promedio de 27 $\pm 1,46 \mathrm{~g}$, adquiridas en el Instituto $\mathrm{Na}$ cional de Salud, Lima-Perú (INS). Fueron mantenidas en jaulas individuales a temperatura ambiente de $22 \pm 2^{\circ} \mathrm{C}$, con ciclo luz/oscuridad de 12 horas y agua ad líbitum.

La aclimatación duró 5 días, el periodo de inducción 4 semanas y el periodo de tratamiento 3 semanas. Posterior a la aclimatación, fueron distribuidas aleatoriamente en tres grupos de seis ratas cada uno: grupo hierro suficiente (HS), grupo hierro deficiente (HD) y el grupo hierro deficiente - extracto $E$. coca (HD-EC). El grupo HS recibió 25 $\mathrm{g} / \mathrm{d}$ de alimento balanceado $(10 \mathrm{mg} / \mathrm{d}$ de hierro elemental), durante 7 semanas. Al grupo HD se le administró 25 $\mathrm{g} / \mathrm{d}$ de dieta ferropénica $(3,41 \mathrm{mg} / \mathrm{d}$ de hierro elemental), durante 7 semanas. El grupo HD-EC recibió $25 \mathrm{~g} / \mathrm{d}$ de dieta ferropénica $(3,41 \mathrm{mg} / \mathrm{d}$ de hierro elemental) durante 7 semanas y, a partir de la semana 5 , se agregó $18 \mathrm{~g} / \mathrm{d}$ de extracto etanólico de E. coca $(30,27 \mathrm{mg} / \mathrm{d}$ de hierro elemental).

La dieta balanceada fue adquirida en el Instituto Nacional de Salud. Para la preparación de la dieta ferropénica, se utilizó $100 \mathrm{~g}$ de leche en polvo, $4 \mathrm{~g}$ de maicena y $2 \mathrm{~g}$ de celulosa, según Carbajal y col ${ }^{(20)}$. Para la preparación del extracto etanólico de E. coca, las hojas de coca fueron adquiridas como micropulverizado en la Empresa Nacional de la Coca (Enaco S.A., Lima-Perú). Doce kilogramos del material vegetal fueron macerados con $56 \mathrm{~L}$ de etanol, a temperatura ambiente. Se decantó, filtró y evaporó el solvente para obtener el extracto. Se realizó la determinación cuantitativa del contenido de hierro a las tres dietas descritas, en el Laboratorio de Análisis Químicos USAQ de la Facultad de Química de la UNMSM, mediante el método de espectrofotometría de absorción atómica.

La anemia ferropénica fue medida en función de las variables peso, talla y $\mathrm{Hb}^{(6)}$. El peso fue registrado diariamente con una balanza electrónica EK5055 de $5 \mathrm{~kg}$ de capacidad. Se midió la talla diariamente con un tallímetro para rata, desde el hocico hasta la cola, con la rata en posición de decúbito ventral. La muestra de sangre para el análisis de la $\mathrm{Hb}$ se obtuvo mediante punción intracardiaca, según el protocolo de The laboratory rat ${ }^{(21)}$. Se consideró anemia ferropénica a los niveles de $\mathrm{Hb}$ inferiores de $11 \mathrm{~g} / \mathrm{dL}^{(21)}$.

Se siguió la guía de ética para animales de experimentación Guide for the Care and Use of Laboratory Animals ${ }^{(22)}$.

Se resumió los datos en medias y desviaciones estándar y se utilizó el gráfico de cajas como representación gráfica. Se realizó el análisis con estadística no paramétrica (prueba U de Mann Whitney) y se aceptó como nivel de significancia un valor $p<0,05$. Se analizó los valores de $\mathrm{Hb}$ al término del periodo de inducción entre los grupos HD con HD-EC, y al término del periodo de tratamiento entre los grupos HD con HD. EC, y HD-EC con HS. Para el análisis de las variables peso y talla se analizó el grupo HD con HD-EC al término del periodo de inducción y al término del periodo de tratamiento.

\section{RESULTADOS}

Al final del periodo de inducción, los valores de $\mathrm{Hb}$ fueron 13,4 $\pm 0,1 \mathrm{~g} / \mathrm{dL}$ en el grupo $\mathrm{HS}, 7,4 \pm 1,4 \mathrm{~g} / \mathrm{dL}$ en el grupo $\mathrm{HD}$, y $7,6 \pm 0,6 \mathrm{~g} / \mathrm{dL}$ en el grupo HD. EC. Al final del periodo de inducción, no se encontró diferencia significativa en los niveles de $\mathrm{Hb}$ entre los grupos HD y HD-EC (prueba de U de Mann Whitney; $p=1,00$ ). En ambos grupos 


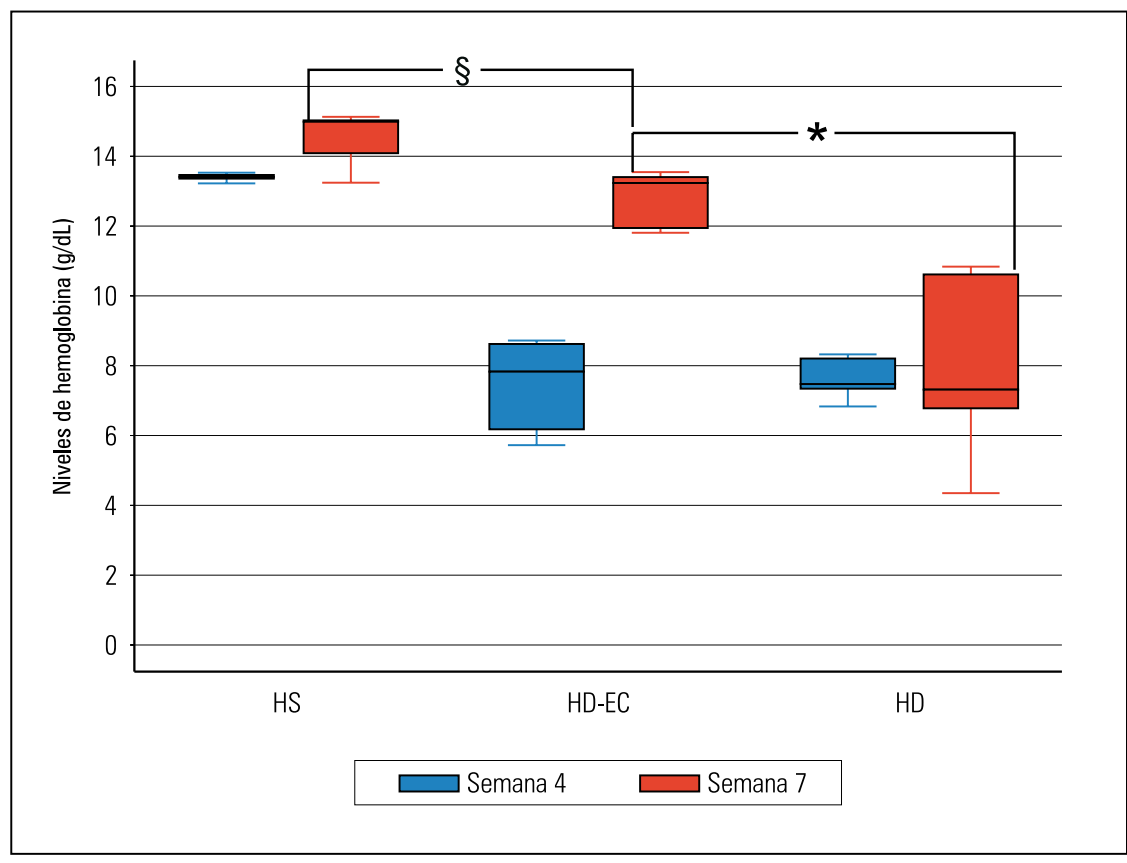

Figura 1. Niveles de hemoglobina al final del periodo de inducción y tratamiento. Semana 4: Al final del periodo de inducción. Semana 7: Al final del periodo de tratamiento. $\left(^{*}\right)$ y $(\S)$ indican diferencia significativa.

se obtuvo valores de $\mathrm{Hb}$ menores de $11 \mathrm{~g} / \mathrm{dL}$, con un valor máximo de $8,7 \mathrm{~g} /$ dL y mínimo de $5,7 \mathrm{~g} / \mathrm{dL}$ (figura 1 ).

Al término del periodo de tratamiento, los valores de $\mathrm{Hb}$ fueron $14,6 \pm 0,9$ $\mathrm{g} / \mathrm{dL}$ en el grupo HS, $7,8 \pm 2,5 \mathrm{~g} / \mathrm{dL}$ en el grupo $\mathrm{HD}$, y $12,8 \pm 0,8 \mathrm{~g} / \mathrm{dL}$ en el grupo HD-EC. Se encontró diferencia significativa entre los valores de $\mathrm{Hb}$ en los grupos HD y HD-EC (prueba de U de Mann Whitney; $p=0,0062$ ). No se encontró diferencia significativa entre el grupo HD-EC y HS (prueba de U de Mann Whitney; $\mathrm{p}=0,06$ ) (figura 1).

$\mathrm{Al}$ comparar los niveles de $\mathrm{Hb}$ antes y después del tratamiento en cada grupo, se observó diferencia significativa solo en el grupo HD-EC (prueba de Wilcoxon; $p=0,04$ para el grupo HD-EC; $p=0,09$ para el grupo HS; $y$, $p=0,92$ para el grupo HD).

Con respecto al peso, al final del periodo de inducción no se evidenció diferencia significativa entre el grupo HD y HD-EC (prueba de U de Mann Whitney; $p=0,92)$. Al término del periodo de tratamiento no se halló dife- rencia significativa entre el grupo HD y HD-EC (prueba de U Mann Whitney; $\mathrm{p}=0,20$ ).

En el análisis de la talla, después de la inducción, no se encontró diferencia significativa entre el grupo HD y HD. EC (prueba de U de Mann Whitney; $\mathrm{p}=0,40)$. Posterior al periodo de tratamiento, no se halló diferencia significativa entre ambos grupos (prueba de $\mathrm{U}$ de Mann Whitney; $p=0,23$ ).

\section{DISCUSIÓN}

Se encontró que la administración de $18 \mathrm{~g} / \mathrm{d}$ de E. coca añadido a la dieta ferropénica incrementó los niveles de hemoglobina hasta alcanzar valores normales. El preparado de E. coca presenta alto contenido de hierro elemental $(30,27 \mathrm{mg} / \mathrm{d})$, el cual es necesario para la síntesis de hemoglobina. Se conoce que el hierro no hem, encontrado en los vegetales, es de menor biodisponibilidad que el hierro hem. Además los fitatos, presentes en los vegetales, inhiben la absorción intestinal de hierro por su actividad quelante. Sin embargo, se plantea que la presencia de vitamina C en E. coca favorecería la absorción de hierro. La vitamina $C$ promueve la transformación de $\mathrm{Fe} 3+$ a $\mathrm{Fe} 2+$ y mejora su absorción ${ }^{(23)}$. Además, interfiere en la actividad quelante de hierro de los fitatos ${ }^{(24)}$.

Siendo E. coca un producto vegetal, presenta alto contenido de fibra. Se considera que la fibra impide la absorción de hierro a nivel intestinal ${ }^{(25)}$. Sin embargo, Feltrin señala que la fibra soluble de pectina no impide la absorción intestinal de hierro ${ }^{(26)}$. Además, Weber informa que la administración de una mixtura de fibras (polisacárido de soya, inulina, almidón resistente, goma arábica, fruto-oligosacárido y celulosa) en ratas inducidas a anemia ferropénica que recibieron tratamiento con citrato férrico (30 mg de hierro elemental), no interfirió en la absorción intestinal de hierro, no habiendo diferencia significativa con los resultados del grupo que no recibió fibras ${ }^{(27)}$.

Al finalizar el tratamiento, los pesos del grupo tratado con E. coca no difirieron significativamente con los pesos del grupo no tratado, a diferencia de García y col., quienes evaluaron la recuperación de la desnutrición moderada en ratas y encontraron que el peso del grupo tratado superó significativamente al peso del grupo desnutrido luego del tratamiento ${ }^{(28)}$.

No hubo diferencia entre la talla del grupo tratado con E. coca y el grupo no tratado. Similar resultado informan García y col., quienes no encontraron diferencias de la talla entre el grupo tratado y el grupo control ${ }^{(28)}$.

La talla y el peso del grupo tratado fueron inferiores a los valores del grupo no inducido a anemia ferropénica. Se propone que los estados nutricionales deficientes de hierro en edades tempranas pueden producir daño irreversible en el crecimiento y desarrollo, evidenciado en alteraciones de peso y talla, pese a recuperar los niveles normales de hemoglobina ${ }^{(29)}$. 
Si bien no se realizó análisis bioquímicos que determinaran el carácter ferropénico de la anemia inducida, Kamei y col. (23) demostraron que la anemia inducida por dieta deficiente de hierro producía anemia ferropénica, sustentados por los niveles séricos de hemoglobina, niveles séricos de hierro y niveles de hierro hepático. Además, Catani y col. indujeron anemia ferropénica con una dieta libre de hierro y demostraron el carácter ferropénico de la anemia mediante los niveles de hemoglobina, hematocrito y hierro hepático ${ }^{(25)}$.

No se realizó medición basal de la hemoglobina para evitar el riesgo de muerte que representa el método de extracción en los animales de experimentación por su corta edad. Además, según The Merck Veterinary Manual, la cantidad de sangre a recolectar debería ser menor al $1 \%$ del peso corporal del animal, por lo que $0,27 \mathrm{~mL}$ de sangre hubiera sido insuficiente para el análisis sanguíneo ${ }^{(30)}$.

El presente estudio propone un producto vegetal que contiene hierro y vitamina C, a diferencia de Tillán-Capó y col., quienes utilizaron Cassia grandis como suplemento nutricional para la absorción de hierro ${ }^{(31)}$.

En este estudio, la administración del extracto etanólico de E. coca spp. tuvo un efecto terapéutico sobre la anemia ferropénica inducida en ratas macho Holtzman, sustentado por el incremento en los niveles de hemoglobina mas no por el peso y la talla.

Se sugiere realizar más estudios sobre el potencial rol terapéutico de E. coca en la anemia ferropénica.

\section{AGRADECIMIENTOS}

Al Sr. Alberto Cabezas Gutiérrez y Sr. Ernesto Sonco, técnicos en Laboratorio del Departamento Académico de Ciencias Dinámicas de la Facultad de Medicina de la UNMSM, por las instrucciones brindadas en el manejo de técnicas en animales.

\section{REFERENCIAS BIBLIOGRÁFICAS}

1. WHO/UNICEF. Focusing on anaemia: Towards an integrated approach for effective anaemia control. 2004.

2. McLean E, Cogswell M, Egli I, Wojdyla D, Benoist B. Worldwide prevalence of anaemia, WHO Vitamin and Mineral Nutrition Information System. Public Health Nutr. 2009;12(4):444-54.

3. Mahoney D. Anemia in at-risk populations-what should be our focus? J Clin Nutr. 2008;88(88):1457-58.

4. WHO. Centers for Disease Control and Prevention. Assessing the iron status of populations. Geneva: World Health Organization; 2007. Dsiponible en: http://www.who.int/nutrition/publications/micronutrients/anaemia_iron_deficiency/9789241596107. pdf.

5. Collard K. Iron homeostasis in the neonate. Pediatrics. 2009;123:1208-16.

6. Buchanan G. Screening for iron deficiency during early infancy: is it feasible and is it necessary? Am J Clin Nutr. 2009;89(2):473-74.

7. INEI. Perú. Lactancia y nutrición de niñas, niños y madres. Encuesta Demográfica y de Salud Familiar 2011.Encuesta Demográfica y de Salud Familiar. Disponible en: http://www.inei.gob.pe/biblioineipub/ bancopub/Est/Lib1027/index.html

8. MINSA.PERÚ 2011.Lineamientos de gestión de la estrategia sanitaria de alimentación y nutrición saludable. Situación nacional de la anemia y sus determinantes. (página 27). Disponible en: http:// bvs.minsa.gob.pe/local/MINSA/1821.pdf

9. Ziegler E, Nelson S, Jeter J. Iron status of breastfed infants is improved equally by medicinal iron and ironfortified cereal. Am J Clin Nutr. 2009;90(1):76-87.

10. Duran P. Anemia por deficiencia de hierro: estrategias disponibles y controversias por resolver Iron deficiency anemia: available strategies and controversies to resolve. Arch Argent Pediatr. 2007;105(6):488-90.

11. Rosado J, González K, Caamaño M, Garcia O, Preciado R, Odio M. Efficacy of different strategies to treat anemia in children: a randomized clinical trial. Nutr J. 2010;9:40.

12. Merino V, Lozano D, Torrico F. Factores que influyen la adherencia a la suplementación con sulfato ferroso durante el embarazo. Gac Med Bol. 2010;33(2):21-5.

13. Caraballo D, Volpato G. Plantas medicinales que se venden en el mercado El Rio, Camaguey, Cuba. Revista Mexicana de biodiversidad. 2008;79:24359.

14. Galindo A, Fernández-Alonso JL. Especies cultivadas del género Erythroxylum P. Browne. Revisión del tema desde la perspectiva forense. Rev Cient Instit Nac Med Legal Ciencias Forenses. 2009;1(3):7-15.

15. Penny M, Zavaleta A, Lemay M, Liria M, Huaylinas $M$, Alminger $M$, et al. Can coca leaves contribute to improving the nutritional status of the Andean population? Food Nutrit Bull. 2009;30(3):205-16.

16. Duke J, Aulik D, Plowman T. Nutritional value of coca. Bot Mus Leafl Harv Univ. 1975(24):113-9.

17. Collazos C, Urquieta R, Alvistur E. Nutrición y coqueo. Rev Viernes Médico. 1975;16(1):4.

18. Soberón M, Oriondo R, Estrada E, Arnao I, Cordero $A$, Velásquez L, et al. Impacto de una intervención alimentaria con un concentrado proteico de Medicago sativa $L$ (alfalfa), en niños pre escolares con desnutrición crónica. An Fac med. 2009;70(3):16874.

19. Clardy S, Wang X, Zhao W, Liu W, Chase G, Beard $J$, et al. Acute and chronic effects of developmental iron deficiency on mRNA expression patterns in the brain. J Neural Transmission. 2006:173-96.

20. Carbajal-Urteaga F, Chirinos-Saire J, Durán-Páucar J, Espinal-Papuico A, Luciani-Huaccac L, LucasLimaymanta R. Administración de Amaranthus $s p$ en la dieta de ratas albinas para el tratamiento de anemia ferropénica. Presentación en Jornada de Investigación Experimental Curso de Farmacologia 2000, UNMSM, Perú, 2000.

21. Suckow M, Weisbroth S, Franklin C. The Laboratory Rat. 2nd ed. New York: Elsevier, editor. 2006.

22. Guide for the Care and Use of Laboratory Animals. Eight ed. Washington D.C.: National Academy Press; 2010.

23. Kamei A, Watanabe $Y$, Ishijima T, Uehara M, Arai S, $\mathrm{Kato} \mathrm{H}$, et al. Dietary iron-deficient anemia induces a variety of metabolic changes and even apoptosis in rat liver: a DNA microarray study. Physiol Genomics. 2010;42:149-56.

24. Thankachan P, Walczyk T, Muthayya S, Kurpad AV, Hurrell RF. Iron absorption in young Indian women: the interaction of iron status with the influence of tea and ascorbic acid. Am J Clin Nutr. 2008;87(4):881-6.

25. Catani M, Amâncio OM, Fagundes-Neto U, Morais $\mathrm{MB}$. Dietary cellulose has no effect on the regeneration of hemoglobin in growing rats with iron deficiency anemia. Braz J Med Biol Res. 2003;36(6):693-7.

26. Feltrin C, Batista de Morais M, de Cássia Freitas K, Beninga de Morais T, Fagundes Neto U, Silvério Amancio OM. Effect of soluble fiber pectin on growth and intestinal iron absorption in rats during recovery from iron deficiency anemia. Biol Trace Elem Res. 2009;129(1-3):221-8.

27. Weber TK, Freitas Kde C, Amancio OM, de Morais MB. Effect of dietary fibre mixture on growth and intestinal iron absorption in rats recovering from iron-deficiency anaemia. $\mathrm{Br} \mathrm{J}$ Nutr. 2010;104(10):1471-6.

28. Garcia N, Gámez M, Fernández M, Iduate M.E. Estudio del comportamiento de variables antropométricas en la recuperación nutricional de ratas desnutridas tratadas con BG-400 Revista Ciencias. [revista en la Internet] [Citado Mar 2012 31]. Disponible en : http://www.sabetodo.com/documentos/ antropometricasratasdesnutridas.pdf

29. Merino JM. Anemias en la infancia. Anemia ferropénica. Pediatr Integral. 2004;8(5):385-403.

30. The Merck Veterinary Manual Online [database on the Internet]. 2011. Available from: http://www. merckvetmanual.com/mvm/index.jsp.

31. Tillán Capó J, Rodríguez Chanfrau J, Gómez Mirabal JM, Pardo Ruíz Z, Agüero Fernández S. Actividad antianémica de la Cassia grandis L. Rev Cubana Farm [revista en la Internet]. 2004 Dic [citado 2012 Mar 31] ; 38(3): 1-1. Disponible en: http://scielo. sld.cu/scielo.php?script=sci_arttext\&pid=S0034$75152004000300009 \&$ Ing $=$ es.

Artículo recibido el 31 de marzo de 2012 y aceptado para publicación el 10 de diciembre de 2012

Conflictos de interés:

Los autores declaran no tener conflicto de interés en la publicación del presente artículo.

\section{Correspondencia:}

Elena Carbajal Lázaro

Correo electrónico: minelena3c@hotmail.com 